\title{
Apego y Perdón en el Contexto de las Relaciones de Pareja
}

\section{Attachment and forgiveness in the context of couple relationships}

\author{
Mónica Guzmán \\ Universidad Católica del Norte, Chile \\ María Pía Santelices \\ Pontificia Universidad Católica de Chile, Chile \\ Caterina Trabucco \\ Universidad Católica del Norte, Chile
}

(Rec: 6 junio 2012 / Acept: 28 febrero 2015)

\begin{abstract}
Resumen
La teoría del apego permite comprender diversos aspectos del funcionamiento interpersonal, entre ellos, la capacidad para perdonar. El objetivo de este estudio fue evaluar la asociación entre las dimensiones del apego adulto (ansiedad del abandono y evitación de la intimidad) y el perdón de transgresiones ocurridas en el contexto de relaciones de pareja. Asimismo, se buscó comparar el peso predictivo de ambas dimensiones sobre el perdón. Para ello, 647 estudiantes universitarios completaron medidas de auto-reporte del apego romántico y del perdón. Los análisis con ecuaciones estructurales permiten concluir que tanto la ansiedad como la evitación estuvieron asociadas a menor perdón. Además, se constató que las personas con alta evitación evidencian mayor dificultad para perdonar que aquellas con alta ansiedad.

Palabras clave: Apego, ansiedad del apego, evitación del apego, perdón, relaciones de pareja.
\end{abstract}

\begin{abstract}
Attachment theory allows the understanding of several aspects of interpersonal functioning, including the ability to forgive. The aim of this study was to evaluate the association between adult attachment dimensions (anxiety over abandonment and avoidance of intimacy) and forgiveness of transgressions occurred in romantic relationships. Additionally, it was sought to compare the predictive weight of each dimension on forgiveness. With this purpose, 647 university students completed self-report measures of romantic attachment and forgiveness. Analysis through structural equation modeling allow to conclude that both higher anxiety and/or higher avoidance were associated to lower forgiveness. Additionally, individuals with high levels of avoidance show more difficulties to forgive than those with high levels of anxiety.

Key words: Attachment, anxiety over abandonment, avoidance of intimacy, forgiveness, romantic relationships
\end{abstract}




\section{Introducción}

En la mayor parte de las relaciones de pareja se producen conflictos que pueden tener relación con haberse sentido herido o traicionado por alguna conducta del otro, siendo ejemplo de tales ofensas la crítica o una infidelidad. Una de las estrategias para enfrentar este tipo de situaciones es el perdón, entendido como el descenso en la negatividad de los pensamientos, sentimientos y conductas hacia quien ha cometido la ofensa (Fincham, Hall y Beach, 2006).

La teoría del apego (TA), que es un marco conceptual que permite comprender diversos aspectos del funcionamiento en relaciones cercanas, se ha incorporado al estudio del perdón (e.g., Gassin y Lengel, 2011; Kachadourian, Fincham y Davila, 2004; Lawler-Row, Younger, Piferi y Jones, 2006; Webb, Call, Chickering, Colburn y Heisler, 2006).

Esta teoría, desarrollada por Bowlby $(1969,1979$, 1980), postula que las experiencias vinculares tempranas se internalizan como esquemas representacionales denominados modelos operativos internos de sí mismo y los otros que influirían la forma de enfrentar las relaciones afectivas adultas, especialmente ante situaciones de conflicto o estrés.

En la conceptualización del apego en el adulto, se ha propuesto que dos dimensiones darían cuenta de diferencias individuales en la forma de enfrentar vínculos de intimidad: la ansiedad o temor al abandono y la evitación de la cercanía e intimidad (Mikulincer y Shaver, 2007). Las personas seguras en el apego, experimentarían baja ansiedad y evitación.

Los estudios sobre el perdón desarrollados en el marco de la TA, han concluido que existe asociación entre ambas variables, indicando que la inseguridad en el apego está asociada a mayor dificultad para perdonar ofensas (e.g., Burnette, Davis, Green, Worthington y Bradfield, 2009; Lawler-Row et al., 2006; Webb et al., 2006).

Sin embargo, pese a la consistencia de estos hallazgos, aún es poco lo que se sabe respecto de la asociación entre estas variables en el contexto específico de las relaciones de pareja. Tampoco es claro qué dimensión del apego, ansiedad o evitación, se encuentra más fuertemente asociada al acto de perdonar, dado que las conclusiones en torno a este punto han sido poco consistentes, lo cual dificulta, por ejemplo, la posibilidad de desarrollar intervenciones diferenciales orientadas a la reparación de heridas interpersonales. De este modo, el objetivo principal de este estudio fue evaluar un modelo acerca de la asociación entre ambas variables en el contexto de transgresiones ocurridas en relaciones de pareja, considerando como variables predictoras ambas dimensiones del apego. Adicionalmente, se buscó examinar qué dimensión del apego, ansiedad o evitación, se encuentra más fuertemente asociada al perdón.

\section{Apego Adulto}

La TA, cuya formulación fue iniciada por John Bowlby $(1969,1979,1980)$ propone que los seres humanos están innatamente motivados a establecer vínculos afectivos hacia los cuales recurrir en momentos de necesidad. Si bien inicialmente esta teoría fue discutida en el ámbito de las relaciones tempranas, surgió con posterioridad una serie de estudios que han aplicado los principios del apego para la comprensión de las relaciones de pareja (Hazan y Shaver, 1987), por ser estos vínculos que propician la intimidad y el cuidado mutuo (Fraley y Shaver, 2000).

En el adulto, el apego ha sido descrito en función de dos dimensiones: ansiedad y evitación. La dimensión de ansiedad alude al grado en que la persona se siente segura o no respecto de la disponibilidad del otro y el nivel en que experimenta preocupación por el potencial abandono o rechazo. Por su parte, la dimensión de evitación alude al grado en que la persona se siente cómoda con la cercanía y dependencia de otros. Se presume que las personas que tienen baja ansiedad y baja evitación serían seguras en el apego, por cuanto se perciben a sí mismas como merecedoras de cuidado y no temen involucrarse íntimamente con otro. Se ha propuesto además que de la combinación de ambas dimensiones, se configuran los estilos de apego (Bartholomew y Horowitz, 1991), entendidos como patrones sistemáticos de expectativas, estrategias de regulación emocional y conducta social (Feeney y Noller, 2001). Batholomew y Horowitz (1991), desarrollaron un modelo de cuatro categorías de apego, uno seguro y tres inseguros: preocupado, temeroso, desentendido, a los cuales subyacen las dos dimensiones descritas que han sido asociadas a distintos aspectos del funcionamiento interpersonal (Mikulincer, Shaver y Slav, 2006).

De la descripción anterior se desprende que la medición del apego puede hacerse tanto categorial (estilos de apego) como dimensionalmente (ansiedad y evitación) (Martínez y Santelices, 2005).

Los estudios que han aplicado el apego a las relaciones románticas muestran que éste se manifiesta, entre otros aspectos, en las estrategias de resolución de conflictos y regulación del afecto ante situaciones de amenaza (Feeney y Noller, 2001; Mikulincer, Shaver y Pereg, 2003). Así, las personas seguras en el apego utilizan la búsqueda de proximidad y apoyo como estrategia para afrontar el estrés o situaciones de conflicto, la llamada estrategia primaria, 
fundamentada en una imagen positiva de sí mismo y en la confianza en la disponibilidad del otro para proveer consuelo (Mikulincer et al., 2003). En cambio, las personas con alta ansiedad y/o evitación, poseen representaciones frustrantes de la figuras de apego, que las conduce a ocupar las denominadas estrategias secundarias del apego ante el estrés: hiperactivación o desactivación (Mikulincer et al., 2006). Las personas con alta ansiedad ocupan estrategias de hiperactivación, que se manifiestan en esfuerzos por lograr la atención, apoyo y amor del otro, una conciencia constante de sus sentimientos negativos, acompañada al mismo tiempo de inseguridad respecto de la posibilidad de conseguirlos (Collins y Read, 1990). En cambio, las personas con alta evitación tienden a ocupar estrategias de desactivación, que se manifiestan en la negación de las necesidades emocionales, la mantención de la distancia emocional y la excesiva confianza en sí mismos como fuente de protección (Mikulincer et al., 2003). De este modo, cuando la pareja se constituye en fuente del dolor emocional, como ante una transgresión, es probable que se activen estos mecanismos de regulación, los que podrían tener incidencia sobre la facilidad o disposición para perdonar.

\section{Perdón}

El perdón y las disculpas son fenómenos encontrados en todas las relaciones humanas y por extensión también en los vínculos románticos. Partiendo desde un interés circunscrito al ámbito religioso y filosófico, la motivación por el estudio del perdón se ha ido moviendo progresivamente al área de la psicología (para una revisión del tema, ver Guzmán, 2010b).

El número de definiciones del perdón en la literatura científica es amplio. Así por ejemplo, Makinen y Johnson (2006) señalan que el perdón involucra reparar heridas emocionales, restaurar la confianza y reconstruir el vínculo. Por su parte, Gordon y Baucom (2003), sostienen que el perdón implica el logro de una visión más balanceada de la relación, la disminución de los sentimientos negativos hacia el otro y un menor deseo de castigarlo. Finalmente, McCullough, Worthington y Rachal (1997), conciben el perdón como una transformación motivacional en dos componentes: la motivación a evitar a la persona que ha ofendido y la motivación a buscar venganza, señalando que el perdón ocurriría cuando ambas motivaciones disminuyen. Pese a la diversidad de énfasis de estas definiciones, la mayoría de los autores coincide en considerar que el perdón implica un descenso en la negatividad de los pensamientos, sentimientos y conductas hacia el ofensor (Fincham, Hall y Beach, 2006).
Además de este aspecto, los autores coinciden en que el perdón puede ser entendido de varias maneras: como un estado, una característica de ciertas unidades sociales o una característica de personalidad (McCullough y Witvliet, 2002). El perdón episódico o como estado, se refiere al perdón de una ofensa en particular (McCullough et al., 1997; Paleari, Regalia y Fincham, 2009). El perdón diádico es entendido como la disposición a perdonar dentro de una relación específica (Kachadourian et al., 2004). Finalmente, el perdón disposicional alude a la tendencia a perdonar a través del tiempo y las situaciones (Berry, Worthington Jr., Parrott, O'Connor y Wade, 2001; Thompson et al., 2005).

En el presente estudio se ocupó la definición de McCullouhg et al. (1997), considerándose que la persona perdona a otra cuando logra liberarse del resentimiento hacia quien la ha herido, lo que se traduce en una menor motivación a distanciarse y vengarse de éste. Además, se ha optado por estudiar el perdón episódico por ser menos permeable a la deseabilidad social que el reporte de la tendencia general a perdonar. Además, interesa conocer cómo es que las personas han enfrentado transgresiones que han vivido efectivamente, más que cómo enfrentarían situaciones hipotéticas. Tal decisión se fundamenta en que la tendencia a perdonar no siempre predice el perdón de una ofensa real (e.g., Kachadourian et al., 2004).

\section{Apego y Perdón}

La TA ofrece un marco conceptual para comprender el perdón. Primero, porque los temas vinculados al apego se activan en situaciones de amenaza, como puede ser la ocurrencia de una transgresión (Kobak y Sceery, 1988), siendo probable que afecten la valoración de la ofensa, de quien ofende y de sí mismo como receptor de ella. Segundo, porque los estilos de apego reflejan la habilidad para regular emociones negativas, que son las que se requiere manejar ante una herida interpersonal (Webb et al., 2006). Como resultado de ello, sería esperable que las personas con mayor seguridad en el apego manejaran mejor la amenaza representada por una transgresión y, por lo tanto, estuviesen más inclinadas a perdonar. Al respecto, existe una serie de estudios que han sido consistentes en confirmar la relación entre ambas variables, tanto si el perdón es concebido como un estado (e.g., Davidson, 2000; Lawler-Row et al., 2006) o como un rasgo (e.g., Webb et al., 2006).

Uno de los primeros estudios sobre el tema reportó que la seguridad del apego estaba vinculada a mayores niveles de perdón (Davidson, 2000). A similares resultados llegó Luebbert (2000) quien además concluyó que las personas 
con estilos preocupados tendían a forzar el perdón prematuramente (pseudoperdón), sin realizar un procesamiento adecuado de la herida, a diferencia de las personas con estilos de apego seguro que las elaborarían a un nivel más profundo. Por su parte, Blount-Mathews (2004) concluyó que las personas con apego seguro eran las que reportaban los niveles más altos de perdón hacia sus padres y aquellas con estilos de apego preocupado los más bajos. Contrariamente a los resultados del estudio de Luebbert (2000), se encontró que las personas con estilos de apego evitativo eran las que tendían al pseudoperdón.

Otro estudio, conducido por Webb et al. (2006), reportaron que la seguridad del apego predecía mayor disposición a perdonar en general, a sí mismo, a otros y a una situación, mientras que para los apegos preocupados hubo una asociación inversa con el perdón en estas tres dimensiones. En el caso de los patrones evitativos, sólo hubo una asociación inversa con el perdón de otros y no con las otras dimensiones. Del mismo modo, Lawler-Row et al. (2006) evaluaron la relación entre apego y el perdón tanto episódico como disposicional. Una vez más, se concluyó que las personas con apego seguro tendían a perdonar más que las inseguras (tanto en general como una ofensa específica), mostrando menor tendencia a evitar al ofensor. Finalmente, Wang (2008), concluyó que bajos niveles de ansiedad y altos de cercanía estuvieron asociados a mayor perdón disposicional.

Los pocos estudios que han vinculado estas variables en el contexto de relaciones de pareja han mostrado resultados similares. Kachadourian et al. (2004), concluyeron que tener un modelo positivo de sí mismo y del otro estaba asociado a mayor perdón. Mikulincer et al. (2006), realizaron un estudio con estudiantes universitarios y otro con matrimonios. En el primero se concluyó que ambas dimensiones del apego hicieron contribuciones únicas al perdón disposicional y episódico, más allá de sus asociaciones con la autoestima y la confianza. La evitación del apego estuvo inversamente asociada a la disposición a perdonar y directamente a la motivación hacia la venganza y la evitación de la pareja. La ansiedad del apego no estuvo asociada a la disposición a perdonar, pero sí a vivir el perdón con una menor sensación de bienestar. En el estudio con matrimonios, se examinaron fluctuaciones diarias en perdonar al cónyuge de acuerdo a los comportamientos de éste. Las personas con alta ansiedad y/o evitación mostraron bajo niveles de perdón aún en días en que percibieron a sus cónyuges como atentos.

Por su parte, Finkel, Burnette y Scissors (2007) reportaron que la inducción del estado de ansiedad del apego, interactuaba con las creencias acerca de la relación (si estaba destinada a funcionar o no) en predecir el perdón. Creencias fuertes sobre el destino predecían menor perdón para personas que estaban experimentando un estado de ansiedad del apego, pero no en quienes estaban experimentando un estado de seguridad. Finalmente, Burnette, Taylor, Wothington y Forsyth (2007) encontraron que el apego estaba asociado al perdón disposicional mediado por la tendencia a rumiar acerca de la transgresión. Ampliando estos resultados, Burnette et al. (2009), concluyeron que la relación entre apego y perdón estuvo mediada parcialmente por la tendencia a rumiar en personas con alta ansiedad y completamente por la baja empatía en personas con alta evitación.

En síntesis, el conjunto de los estudios expuestos son coincidentes en confirmar la asociación entre apego y perdón. Sin embargo, son escasas aún las investigaciones que han abordado este fenómeno en el marco de las relaciones de pareja. Además, la gran mayoría de las investigaciones en torno al perdón en relaciones románticas lo han hecho focalizándose en relaciones de pareja en curso; esto tiene como falencia que sesga la muestra hacia quienes con mayor probabilidad han perdonado una transgresión, dada la correlación entre perdón y reconciliación (Hall y Fincham, 2006) o a favor de eventos hirientes menos severos que han permitido la continuidad de la relación. Este estudio, cuyo foco es la perspectiva individual, pretende subsanar este aspecto, al considerar tanto personas que reportaron su grado de perdón respecto de una pareja actual, como quienes lo hicieron en base a una relación ya disuelta.

Finalmente, pocos estudios han comparado directamente el valor predictivo de las dimensiones del apego sobre el perdón. Con evaluaciones categoriales del apego, algunos han concluido que son los apegos preocupados (con alta ansiedad) los que perdonan menos (e.g., Blount-Mathews, 2004; Wang, 2008), mientras que otros proponen que son los patrones evitativos (e.g., Gassin y Lengel, 2011; Kachadourian et al., 2004; Mikulincer et al., 2006). Esta investigación pretende responder de manera específica esta interrogante, comparando el peso predictivo de cada dimensión del apego. Se propone que la evitación del apego tendrá una asociación más fuerte con el perdón, hipótesis fundamentada en que es el modelo del otro el que parece subyacer a las diferencias en las evaluaciones que hacen las personas de los conflictos con sus parejas (Mikulincer et al., 2006), de las expectativas respecto al otro y de la interpretación de sus acciones. En cambio, en las personas con alta ansiedad, la actitud hacia el perdón tendería a ser más ambivalente, pues hay dos fuerzas en conflicto: por un lado está la tendencia a rumiar (Burnette et al., 2009) y a intensificar las emociones negativas asociadas a una herida interpersona, pero por otro, está también el temor a 


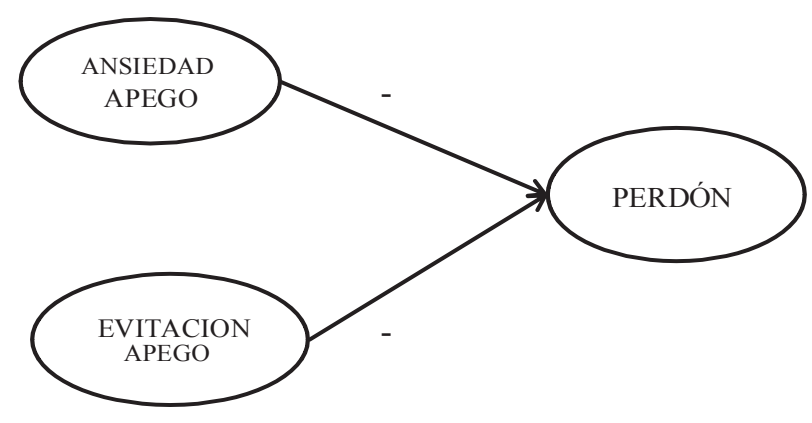

Figura 1.

Modelo Estructural Hipotetizado

la separación, por lo que su relación con el perdón puede ser más ambigua y por lo tanto, menor en magnitud.

Considerando los antecedentes expuestos, el objetivo del estudio fue evaluar un modelo acerca de la asociación directa entre las dimensiones del apego y el perdón en relaciones de pareja y secundariamente, comparar el valor predictivo de dichas dimensiones en su efecto sobre el perdón. Específicamente, se predijo que a mayor ansiedad y/o evitación estarían asociados a menores niveles de perdón (entendido como baja motivación hacia la venganza y a la evitación del otro) (ver Figura 1). Además se hipotetizó que la dimensión de evitación estaría más fuertemente asociada al perdón que la dimensión de ansiedad.

\section{Método}

\section{Diseño de Investigación}

Se realizó un estudio de tipo transversal, con un diseño correlacional no experimental, basado en un cuestionario de auto-reporte contestado en forma individual por cada participante, en el que se testeó un modelo que consideró como variable endógena el perdón (con dos dimensiones, motivación hacia la venganza y motivación hacia la evitación del ofensor) y como variables exógenas la ansiedad y evitación del apego.

\section{Participantes}

Se evaluó una muestra no probabilística de conveniencia, no clínica, compuesta por 647 estudiantes universitarios provenientes de 7 universidades de la ciudad de Santiago y 3 de Antofagasta. Los criterios de inclusión fueron tener más de 18 años y que tuviesen o hubiesen tenido, al momento de la evaluación, una relación de pareja de al menos 6 meses de duración. Se estableció este tiempo como mínimo por ser el que se considera necesario para que se desarrolle un cierto sentido de interdependencia e identidad como pareja (Fincham, Jackson y Beach, 2005).

Del total de la muestra $(N=647), 395$ fueron mujeres y 252 hombres, con edades fluctuantes entre 18 y 45 años y un promedio de 21.2 años $(D S=3.94)$. Un $97.8 \%$ de los participantes reportaron ser solteros, un $1.2 \%$ estaba casados y un $1 \%$ separados o divorciados.

A los participantes se les pidió responder pensando en aquella pareja que hubiese sido más significativa para ellos: $324(50.1 \%)$ de ellos contestó basándose en una relación de pareja actual, mientras que el $49.9 \%$ restante $(n=323)$ lo hizo con base en una pasada.

\section{Instrumentos}

Además de la caracterización sociodemográfica, se aplicaron los siguientes instrumentos de auto-reporte para evaluar las dimensiones del apego y el perdón episódico:

Experiences in Close Relationships (ECR, Brennan, Clark y Shaver, 1998), que es un cuestionario de 36 ítems en formato Likert $(1=$ No me representa para nada, $7=$ Me representa completamente), que arroja puntajes en dos escalas ortogonales de 18 ítems cada una: la ansiedad y la evitación asociada al apego. La escala de ansiedad incluye ítems tales como "Necesito que mi pareja me confirme constantemente que me quiere", mientras que la escala de evitación incluye ítems como "Me cuesta depender emocionalmente de una pareja".

Además de la obtención de un puntaje asociado a cada dimensión, el ECR permite ubicar a las personas en una de cuatro categorías (seguro, preocupado, temeroso, evitativo). Dado el objetivo del estudio, en este caso se utilizó la evaluación dimensional. Puntajes más altos indican mayor grado de evitación $\mathrm{y} / \mathrm{o}$ ansiedad, por lo tanto mayor inseguridad en el apego.

Este instrumento se escogió porque ha sido utilizado ampliamente en la investigación del apego adulto y ha mostrado tener buenas propiedades psicométricas. Los índices de confiabilidad, mediante el alpha de Cronbach, son de .91 y .94 para las escalas de ansiedad y evitación respectivamente. Los autores reportaron además que la confiabilidad test-retest fue de .70 para cada subescala (Brennan et al., 1998). La evidencia de validez de constructo del ECR está basada en estudios que muestran que es un predictor significativo de constructos teóricamente relevantes, tales como la satisfacción marital (Brennan et al., 1998) y la 
presencia de problemas interpersonales (Wei, Vogel, Ku y Skalik, 2005), entre otros.

Para este estudio se ocupó una versión adaptada al español por Guzmán (2010a). El alpha de Cronbach ( $N$ $=647$ ) fue de .87 en la escala de ansiedad y .85 en la de evitación. La correlación entre ambas escalas del apego fue baja y no significativa ( $r=.01, p>.01)$, lo que corrobora la independencia de ambas dimensiones.

Transgression-Related Interpersonal Motivations Inventory (TRIM-12, McCullough et al., 1998). Este es un cuestionario de auto-reporte compuesto de 12 ítems en formato Likert de 5 puntos ( $1=$ Totalmente en desacuerdo, 5 $=$ Totalmente de acuerdo), que evalúan las reacciones de la persona ante transgresiones específicas. Se compone de dos escalas: la motivación a buscar venganza hacia el ofensor (ejemplo: "Deseo que le suceda algo malo"), conformada por 5 ítems y la motivación a evitar al ofensor ("Mantengo entre nosotros la mayor distancia posible"), compuesta por 7 ítems. Para este estudio se ocupó una versión con 11 de los 12 ítems originales adaptada al español por Guzmán (2010a). Se eliminó el ítem 8, "Me cuesta ser cariñoso con él/ella", por no ser aplicable para quienes respondieran el instrumento con base en una relación ya disuelta. Puntajes más altos indican mayor motivación hacia la venganza y la evitación, por lo tanto, menor perdón. Para este estudio, y a fin de facilitar la interpretabilidad de los puntajes, los ítems fueron recodificados, de modo tal que mayor puntaje indica menor motivación hacia la venganza y la evitación.

El TRIM-12 tiene buenas propiedades psicométricas, incluyendo una adecuada estabilidad temporal (.86 para la escala de evitación y 90 para la escala de venganza, mediante el alpha de Cronbach) y apropiada validez, demostrada por su asociación con la medición del perdón mediante un ítem único $(p<.01)$. Además, las escalas del TRIM12 han mostrado asociación con variables de la relación, tales como la satisfacción, el compromiso y la cercanía ( $p$ $<.01$ ) (McCullough et al., 1998). Se optó por utilizar este instrumento por haber sido desarrollado sobre una amplia base teórica, poseer buenas propiedades psicométricas y por su extensa utilización en los estudios sobre el perdón; asimismo, permitía evaluar el perdón tanto en personas que respondieron en base a una relación actual o pasada. El alpha de Cronbach para la muestra en estudio $(N=647)$ fue .80 para la escala de venganza y .92 para la escala de evitación.

\section{Procedimiento}

Para el reclutamiento de la muestra, se coordinó con académicos de universidades la visita a alguna de sus clases a fin de invitar a participar a estudiantes que cumplieran los criterios de inclusión. Al hacerlo, se les otorgó información respecto de los objetivos del estudio y la naturaleza de la participación en el mismo. Siguiendo los requerimientos éticos correspondientes, se enfatizó el carácter voluntario y anónimo de su colaboración. Una vez que las personas que accedieron a participar habían firmado el consentimiento informado, se les pidió que respondiesen los instrumentos. En todas las situaciones, se aplicó el cuestionario en papel.

El cuestionario estuvo dividido en dos partes. Primero, los participantes completaron la caracterización socio-demográfica, siguiendo luego con el instrumento que evalúa apego. Para responder a la segunda parte, se solicitó a los participantes que pensaran en una pareja con la que hubiesen tenido una relación de al menos 6 meses. Si habían tenido más de una relación que hubiese durado más de ese tiempo, se les indicó que escogieran aquella pareja que hubiese sido más significativa, para así aumentar la probabilidad que fuese (o hubiese sido) una figura de apego (Hazan y Shaver, 1987). Posteriormente, $\mathrm{y}$ de modo similar a otros estudios realizados acerca del perdón (e.g., Gordon y Baucom, 2003), se les pidió que proporcionaran por escrito una breve descripción de una experiencia importante en la que se hubiesen sentido heridos por dicha pareja. Respecto de tal transgresión, se consignó tiempo transcurrido desde la misma, tiempo de relación al momento de la ofensa, recurrencia y si involucraba a una pareja actual o pasada. A fin de homogenizar la muestra, se controló el efecto del tiempo y de la recurrencia de la transgresión sobre el perdón incluyéndose sólo casos que reportaron una ofensa que hubiese ocurrido hace menos de un año y en una única oportunidad. Con ello, se disminuyó adicionalmente el efecto que el tiempo podría tener sobre la percepción retrospectiva de de la transgresión y su impacto. Finalmente, se completaron los instrumentos que evalúan el perdón.

\section{Análisis de Datos}

Se ocupó el programa SPSS 17.0 para el análisis preliminar de los datos y el programa EQS 6.1 (Bentler, 2005), para evaluar la asociación entre las dimensiones del apego y el perdón, comparando el peso predictivo de ambas.

Preliminarmente, se llevaron a cabo sucesivos Análisis Multivariados de la Varianza (MANOVA), considerando las características socio-demográficas como variables de agrupación y las variables en estudio (dimensiones del apego y motivación hacia la venganza y hacia la evitación) como variables dependientes, para examinar posibles diferencias en ellas. 
Para responder a los objetivos del estudio, se utilizó la técnica de Modelos de Ecuaciones Estructurales (SEM) porque permite evaluar relaciones entre un conjunto de variables de manera simultánea y porque el empleo de variables latentes otorga mayor potencia al estudio y disminuye el error de medición (Streiner, 2006).

La muestra inicial estuvo conformada por 830 participantes. Dados los criterios de inclusión descritos, 152 casos fueron removidos, quedando la muestra conformada por 678 casos preliminarmente. Luego se realizó un examen de los datos a fin de detectar casos con valores ausentes. Se detectaron 24 casos con un número elevado de datos ausentes en alguna de las escalas ( $>8 \%$ ), por lo que se decidió eliminarlos, tras chequear que fuesen datos faltantes al azar (Hair, Anderson, Tatham y Black, 1999). Aquellos casos con datos incompletos en algunas de las respuestas, fueron reemplazados con la media obtenida en la escala correspondiente. Para detectar casos atípicos multivariados y al ocupar la distancia de Mahalanobis como estadístico, se identificaron 7 casos que excedían el valor crítico $(p<.001)$ que fueron eliminados de los análisis (Hair et al.). Así, la muestra final del estudio quedó conformada por 647 casos.

A continuación, se procedió a evaluar dos supuestos que deben ser considerados al ocupar SEM (Hair et al., 1999): normalidad y ausencia de multicolinealidad. La normalidad de los datos se evaluó con los índices de curtosis y asimetría. Sólo la variable motivación hacia la venganza excedía el valor \pm 2 , situación que fue manejada mediante la reexpresión de las variables construyendo parcelas (parcels) para la elaboración del modelo de medición, procedimiento que se detalla más adelante (Hoyle, 1995). A fin de evaluar la multicolinealidad, se examinaron los coeficientes de correlación entre las variables. De acuerdo a Kline (2005), valores por sobre .85 podría indicar multicolinealidad. Las correlaciones significativas fluctuaron entre .08 y $.42(p<$ $.05)$, por lo que no se viola tal supuesto. Hubo asociación significativa entre todas las variables, excepto entre la evitación y ansiedad del apego, lo cual es esperable, pues se ha propuesto que son dimensiones independientes (Barthlomew y Horowitz, 1991).

\section{Resultados}

\section{Análisis descriptivos}

En la Tabla 1 se presenta la estadística descriptiva de las variables evaluadas para la muestra total.

El promedio de duración de la relación de pareja al momento de ocurrida la transgresión fue de 21.9 meses ( $D S$ $=13.5$ ), lo que indica que en general los participantes se refirieron a transgresiones ocurridas en relaciones estables.

Al evaluar variaciones según características sociodemográficas, se encontraron diferencias estadísticamente significativas en el conjunto de variables dependientes entre hombres y mujeres, $\lambda=.95, F(7,639)=4.19, p<.001, \eta 2$ $=.04$. Los análisis univariados muestran que el efecto del sexo fue estadísticamente significativo sólo para la evitación del apego, $F(1,645)=10.69, p<.001$, con los hombres reportando mayor evitación del apego, $M=2.72(D S=.89)$ que las mujeres, $M=2.49(D S=.91)$ y para la evitación del ofensor, $F(1,645)=3.93, p<.05$. Los hombres reportaron más tendencia a evitar a quien los hirió, $M=3.58(D S=$ 1.27), que las mujeres, $M=3.78(D S=1.21)^{1}$. Los tamaños de los efectos según el sexo fueron pequeños (Cohen, 1992).

No hubo diferencias significativas en las variables dependientes según estado civil, $\lambda=.96, F(7,636)=1.08$,

\footnotetext{
Tal como se indicó en el apartado de instrumentos, los puntajes de las escalas del TRIM-12 fueron recodificados, por lo que puntajes más
} altos, indican menor tendencia a evitar.

Tabla 1. Estadística Descriptiva de las Variables

\begin{tabular}{lccc}
\hline & \multicolumn{2}{c}{ Rango } & \\
\cline { 2 - 3 } Variable & Rango original & Rango real & Media $(D S)$ \\
\hline Ansiedad del apego & $1-7$ & $1.11-6.56$ & $3.60(1.02)$ \\
Evitación del apego & $1-7$ & $1.0-5.69$ & $2.58(0.92)$ \\
Evitación TRIM-12 & $1-5$ & $1.0-5.0$ & $3.70(1.24)$ \\
Venganza TRIM-12 & $1-5$ & $1.2-5.0$ & $4.60(0.62)$ \\
Item global perdón & $1-5$ & $1.0-5.0$ & $3.93(1.21)$ \\
\hline
\end{tabular}

Nota. $N=647$ 
$p=.35, \eta 2=.01$, carrera, $\lambda=.89, F(7,632)=1.32, p=$ $.06, \eta 2=.02$, ni universidad, $\lambda=.86, F(7,630)=1.30, p$ $=.06, \eta 2=.02$.

\section{Evaluación del Modelo Propuesto mediante Modelos de Ecuaciones Estructurales}

Para el examen de los objetivos a través de SEM, se ocupó la matriz de covarianzas como input de análisis de los datos y Máxima Verosimilitud como método de estimación. Además, se analizaron datos completos $(N=647)$.

Existen numerosos indicadores de ajuste que proveen información acerca del grado en que la matriz de covarianza observada reproduce el modelo propuesto. Para este estudio, se consideró el estadístico de bondad de ajuste chi cuadrado $\left(\chi^{2}\right)$, junto a los siguientes indicadores adicionales: NFI, NNFI, CFI y RMSEA y el Intervalo de confianza del 90\% para RMSEA (Byrne, 2006; Kline, 2005), dada la sensibilidad del primero al tamaño muestral.

Un paso preliminar fue testear la validez del modelo de medición (Byrne, 2006). Para definir los indicadores de las variables latentes se procedió a construir parcelas, ocupando para ello las recomendaciones de Little, Cunningham, Shahar y Widaman (2002). Las parcelas son composiciones de puntajes que se crean a partir de un set de ítems que son tratados como indicadores continuos de las variables latentes (Kline, 2005). Se decidió conformar parcelas por dos motivos. Primero, porque el interés estaba puesto en el modelo estructural más que en examinar las relaciones exactas entre los ítems individuales y las variables medidas. Segundo, porque el uso de parcelas aumenta la probabilidad de cumplir el supuesto de normalidad, que es requisito para el uso de la Máxima Verosimilitud (Hoyle, 1995). A ello se suma el logro de una mayor confiabilidad (Kishton y Widaman, 1994). Para las escalas unidimensionales (ansiedad y evitación), las parcelas se formaron asignando aleatoriamente los ítems a cada escala (Kishton y Widaman, 1994). En el caso de la escala de perdón, que tiene más de una dimensión, se usó la aproximación de dominio representativo sugerida por Little et al. (2002), en la cual los indicadores de cada parcela se crearon de tal modo que incluyese una combinación de ítems de cada escala. De este modo, el modelo de medición estuvo conformado por 3 variables latentes y 9 variables observadas. Al especificar el modelo, no se permitió la correlación entre los factores latentes y se fijó en 1 la carga de una de las parcelas en cada factor.

La adecuación del modelo propuesto para explicar la matriz de covarianza observada se evaluó inicialmente mediante el estadístico de bondad de ajuste chi-cuadrado, que arrojó un valor de $\chi^{2}(27, N=647)=80.07, p<.05$, que indicaría discrepancia entre el modelo propuesto y los datos. Sin embargo, dada su sensibilidad al tamaño muestral, se revisaron los otros indicadores de ajuste: $\mathrm{NFI}=.97, \mathrm{NNFI}=$ $.97, \mathrm{CFI}=.97, \mathrm{RMSEA}=.03, \mathrm{IC} 90 \%[.01, .04]$, los cuales denotan un excelente ajuste del modelo a los datos. Además, los parámetros individuales son apropiados en términos de su magnitud y significación estadística, con valores que fluctúan entre .51 y .97 , que superan el valor mínimo de .40 considerado aceptable (Ford, MacCallum y Trait, 1986). En conclusión, el modelo de medición propuesto representa adecuadamente los constructos latentes.

Una vez examinado el modelo de medición, se evaluó el modelo estructural hipotetizado, que posee 2 variables exógenas (ansiedad y evitación del apego) con efectos directos sobre una variable endógena (perdón). Los indicadores de ajuste mediante el $\chi 2(25, N=647))=36.50, p<.05$ sugieren que el modelo no es adecuado. Sin embargo, los tres indices, NFI $=.99$, NNFI $=.99$ y CFI $=.99$, exceden el valor de .95 considerado apropiado, sugiriendo que el modelo hipotetizado evidencia un buen ajuste a los datos. Además, el valor del RMSEA para el modelo es .03, 90\% $\mathrm{IC},[.00, .04)$, representando un buen nivel de precisión. Adicionalmente, la proporción chi cuadrado/gl $=1.45$, se considera apropiada. Al evaluar la significación de los parámetros individuales, ambos fueron significativos ( $p<$ .05 ) y en las direcciones esperadas (ver Figura 2). El modelo explica un 13,2\% de la varianza en el perdón.

En segundo lugar y para evaluar el peso predictivo de ambas dimensiones sobre el perdón, se procedió a comparar dos modelos. En el primero se restringió a ser iguales las cargas de la evitación y ansiedad del apego sobre el perdón, $\chi^{2}(26)=54.09, p<.05$, y en el segundo las cargas de la ansiedad y la evitación del apego se estimaron libremente (ver Figura 8), $\chi^{2}(25)=36.50, p<.05$ Ambos modelos se compararon mediante la diferencia en el chi cuadrado.

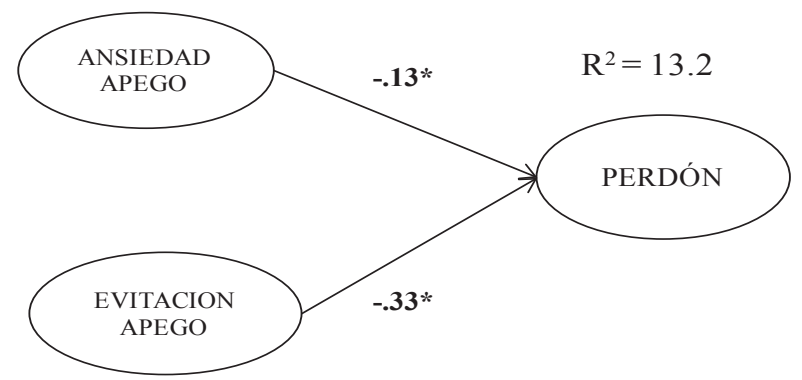

Figura 2. Modelo Estructural

Nota. Todos los parámetros presentados son significativos $(p<$ .05) y corresponden a la solución estandarizada simple. 
La diferencia en el chi cuadrado fue de $\Delta \chi^{2}(1)=15.59$, $p<.05$. El valor crítico para un chi cuadrado con un grado de libertad es de $\chi^{2}=3.48, p=.05$, lo que indica que ambos modelos difieren significativamente. Esto permite concluir que, tal como se predijo, la evitación del apego es una variable que predice con mayor fuerza el perdón que la ansiedad.

\section{Discusión}

La mayor parte de la investigación sobre apego y perdón ha concluido que quienes evidencian mayor seguridad en el apego perdonan más fácilmente que quienes manifiestan menos seguridad (e.g., Burnette, et al., 2009; Mikulincer et al., 2006). Este estudio, además de confirmar la asociación de ambas dimensiones del apego y el perdón, ayuda a clarificar cuál de ellas lo predice con mayor fuerza en el contexto de las relaciones de pareja entre jóvenes: la evitación.

En el caso de la asociación entre el componente de ansiedad y las dos dimensiones del perdón evaluadas (búsqueda de venganza y evitación del ofensor), la relación inversa entre ambas se explicaría a partir del modelo negativo de sí mismo que tienen asociadas las personas con alta ansiedad, que las hace hipersensibles al rechazo y a experimentar intensa rabia ante una transgresión, lo que podría favorecer la presencia de un sesgo atribucional hostil (Mikulincer, 1998). Por su parte, en el caso de la evitación, se ha visto que las personas con alta evitación tienen una imagen negativa del otro, acompañada de un sentido de poca confianza en las buenas intenciones de los demás. La ocurrencia de una transgresión no haría sino confirmar tal presunción, favoreciendo la devaluación de la relación. Del mismo modo, su tendencia a negar sus deseos de intimidad y a evitar la búsqueda de apoyo, generan un espacio menos propicio para obtener gratificación en una relación (Bartholomew y Horotwitz, 1991; Brennan et al., 1998).

Respecto de la comparación del peso predictivo de las dimensiones del apego sobre el perdón, estudios previos habían reportado resultados poco consistentes (e.g., Luebbert, 2000; Blount-Mathews, 2004). Este estudio evaluó explícitamente esta interrogante, reteniéndose la hipótesis que sería la dimensión de evitación la que tiene un peso predictivo mayor.

Este hallazgo puede explicarse a partir de las estrategias de desactivación propias de las personas con alta evitación y del modelo negativo del otro que suele subyacer en ellas (Mikulincer et al., 2006). Específicamente, las personas con alta evitación tienden a maximizar la distancia de sus parejas cuando se enfrentan a situaciones de estrés. Además, suelen ser poco inclinadas a expresar sus necesidades de apoyo, y al no hacerlo, dan poco espacio a la contención del otro y a que éste pueda implementar actos reparatorios ante una transgresión. Adicionalmente, se ha propuesto que las personas con alta evitación tienden a esperar que los demás actúen de manera descuidada y a responder culpando y alejándose del otro ante los conflictos (Pietromonaco, Greenwood y Feldman, 2004).

En contraste, es posible que el menor poder predictivo de la ansiedad se explique a partir de la actitud más ambivalente hacia el perdón. Por un lado, es probable que el intenso temor al abandono las conduzca a expresar perdón a la pareja en un esfuerzo por mantener la proximidad de ésta (pseudoperdón). Sin embargo, al mismo tiempo, las personas con alta ansiedad tienden a rumiar continuamente acerca de las transgresiones (Burnette et al., 2007, Burnette et al., 2009) y a intensificar las emociones negativas asociadas a ellas (Feeney, 2004), lo que impide el perdón genuino. Dada la presencia de estas fuerzas en conflicto, sería esperable que la relación entre ansiedad y perdón sea más débil. En apoyo a esta noción se ha visto, por ejemplo, que en las personas con alta ansiedad la vivencia del perdón es menos placentera (Mikulincer et al., 2006).

A estos elementos teóricos que pueden dar cuenta de este resultado es posible agregar un tópico de corte metodológico: uno de los aspectos evaluados por el TRIM-12 es precisamente la tendencia a distanciarse de quien ha cometido la ofensa, por lo tanto, es posible esperar que en las personas con alta evitación del apego tal asociación sea más fuerte, puesto que se exacerba una tendencia a la evasión que ya se posee, haciéndose más saliente ante una transgresión.

En definitiva, mientras más incómoda se sienta la persona con la cercanía y la intimidad, más difícil le será perdonar. Para ellas es probable que la ocurrencia de una transgresión sea una confirmación que el otro no está disponible, minando aún más la confianza en el vínculo, lo cual podría transformarse en un circuito auto-perpetuante.

Desde el punto de vista clínico, la comprensión del perdón en el marco de la teoría del apego provee información relevante para abordar psicoterapéuticamente el tema del perdón al interior de la pareja, tomando en cuenta las diferencias individuales. Es decir, los resultados del presente estudio relevan la importancia de tomar en cuenta los estilos de apego predominantes en el abordaje clínico de las transgresiones relacionales, puesto que permiten comprender las posibles dificultades en resolver estas transgresiones, que se explican diferencialmente desde los modelos de apego individuales.

Ahora bien, pese al aporte de estos hallazgos en un tema escasamente abordado en el contexto nacional, la presente 
investigación tiene ciertas limitaciones que ofrecen, al mismo tiempo, ideas de investigación futura.

En primer lugar, es importante ser cautos respecto de la generalización de estos resultados a matrimonios y relaciones de convivencia, que sólo representan un 1.2\% de la muestra. Investigación futura pudiese incluir muestras con otro tipo de relaciones y de distintos grupos etáreos. En segundo lugar, los datos obtenidos se basan en instrumentos de auto-reporte, los que si bien son adecuados para evaluar constructos subjetivos, tienen asociados ciertos sesgos, como la deseabilidad social. Además, responder este tipo de medidas requiere tener alta conciencia de sí mismos. En el futuro, complementar el uso de instrumentos de auto-reporte con otro tipo de medidas, por ejemplo datos observacionales, permitiría enriquecer estos hallazgos. Por último, el TRIM12 (McCullough et al., 1998) si bien evalúa dos aspectos importantes del perdón (motivación a evitar y vengarse del ofensor), sería enriquecedor integrar otros instrumentos que evalúen una gama más amplia de los aspectos involucrados en el perdón (e.g., benevolencia). Además, siendo el perdón un fenómeno que se desarrolla en el tiempo, se considera relevante complementar este estudio con otros en que se evalúe el perdón en distintos momentos.

En su conjunto, y pese a las limitaciones de este trabajo, la investigación desarrollada provee una perspectiva distinta para comprender las heridas en relaciones de pareja, integrando la teoría del apego, en un tema escasamente investigado en nuestro país como es el perdón. Así, el perdón es más viable si la relación es importante, si el otro es considerado fuente de protección y si la persona se siente segura en el vínculo de pareja.

\section{Referencias}

Bartholomew, K., y Horowitz, L. M. (1991). Attachment styles among young adults: A test of a four category model. Journal of Personality and Social Psychology, 61, 226-244.

Bentler, P. M. (2005). EQS 6 structural equations program manual. Encino, CA: Multivariate Software (www.mvsoft.com).

Berry, J. W., Worthington Jr., E. L., Parrot, L., O'Connor, L. E., y Wade, N. G. (2001). Dispositional forgivingness: Development and construct validity of the Transgression Narrative Test of Forgivingness (TNTF). Personality and Social Psychology Bulletin, 27, 1277-1290. doi: 10.1177/01461672012710004

Blount-Matthews, K. M. (2004). Attachment and forgiveness in human development: A multi-method approach. (Tesis de Doctorado no publicada), University of California, Berkeley, CA, Estados Unidos.

Bowlby, J. (1969). El vínculo afectivo. Buenos Aires: Paidós.

Bowlby, J. (1979). Vínculos afectivos: Formación, desarrollo y pérdida. Madrid: Morata.

Bowlby, J. (1980). La pérdida afectiva. Buenos Aires: Paidós.

Brennan, K. A., Clark, C. L., y Shaver, P. R. (1998). Self-report measurement of adult attachment: An integrative overview. En J. A. Simpson y W. S. Rholes (Eds.), Attachment Theory and close relationships (pp. 46-76). New York: Guilford Press.

Burnette, J. L., Davis, D. E., Green, J. D., Worthington, E. L., y Bradfield, E. (2009). Insecure attachment and depressive symptoms: The mediating role of rumination, empathy, and forgiveness. Personality and Individual Differences, 46, 276-280. doi: 10.1016/j.paid.2008.10.016

Burnette, J. L., Taylor, K. W., Worthington, E. L., y Forsyth, D. R. (2007). Attachment and trait forgiveness: The mediating role of angry rumination. Personality and Individual Differences, 42, 1585-1596. doi: :10.1016/j.paid.2006.10.033

Byrne, B. M. (2006). Structural equation modeling with EQS, ( $2^{\text {nd }}$ Ed.). New Yersey: Psychology Press.

Cohen, J. (1992). A power primer. Psychological Bulletin, 112, 155-159. doi: 10.1037/0033-2909.112.1.155

Collins, N. L., y Read, S. J. (1990). Adult attachment, working models, and relationship quality in dating couples. Journal of Personality and Social Psychology, 58, 644-663. doi: 10.1037/0022-3514.58.4.644

Davidson, L. L. (2000). Forgiveness and attachment in college students (Tesis de Doctorado no publicada), University of Wyoming, Laramie, WY, Estados Unidos.

Feeney, J., y Noller, P. (2001). Apego adulto. Bilbao: Desclée de Brouwer. Fincham, F. D., Hall, J., y Beach, S. R. (2006). Forgiveness in marriage: Current status and future directions. Family Relations, 55, 415-427. doi: 10.1111/j.1741-3729.2005.callf.x-i1

Fincham, F. D., Jackson, H., y Beach, S. R. H. (2005). Transgression severity and forgiveness: Different moderators for objective and subjective severity. Journal of Social and Clinical Psychology, 24, 860-875. doi: 10.1521/jscp.2005.24.6.860

Finkel, E. J., Burnette, J. L., y Scissors, L. E. (2007). Vengefully ever after: Destiny beliefs, state attachment anxiety and forgiveness. Journal of Personality and Social Psychology, 92, 871-886. doi: : 10.1037/00223514.92.5.871

Ford, J. K., MacCallum, R. C., y Tait, M. (1986). The application of exploratory factor analysis in applied psychology: A critical review and analysis. Personnel Psychology, 39, 291-314. doi: 10.1111/j.17446570.1986.tb00583.x

Fraley, R. C., y Shaver, P. R. (2000). Adult romantic attachment: Theoretical developments, emerging controversies, and unanswered questions. Review of General Psychology, 4, 132-154. doi: 10.1037//10892680.4.2.132

Gassin, E. A., y Lengel, G. J. (2011). Forgiveness and attachment: a link that survives the grave? Journal of Psychology and Theology, 39, 316-329.

Gordon, K. C., y Baucom, D. H. (2003). Forgiveness and marriage: Preliminary support for a measure based on a model of recovery from a marital betrayal. The American Journal of Family Therapy, 31, 179-199. doi: 10.1080/01926180301115

Guzmán, M. (2010a). Apego y perdón de transgresiones relacionales en la pareja: El rol mediador de las atribuciones negativas y la satisfacción (Tesis de Doctorado no publicada), Pontificia Universidad Católica de Chile, Santiago, Chile

Guzmán, M. (2010b). El perdón en relaciones cercanas: Conceptualización desde una perspectiva psicológica e implicancias para la práctica clínica. Psykhe, 19, 19-30. doi: 10.4067/S0718-22282010000100002

Hair, J. F., Anderson, R. E., Tatham, R. L., y Black, W. C. (1999). Análisis Multivariante $\left(5^{a} E d\right.$.). Madrid: Pearson Educación.

Hall, J. H., y Fincham, F. D. (2006). Relationship dissolution following infidelity: The roles of attributions and forgiveness. Journal of Social y Clinical Psychology, 25, 508-522. doi: 10.1521/jscp.2006.25.5.508

Hazan, C., y Shaver, P. (1987). Romantic love conceptualized as an attachment process. Journal of Personality and Social Psychology, 52, 511-524. doi: 10.1037/0022-3514.52.3.511

Hoyle, R. H. (1995). Structural equation modeling. Thousand Oaks: Sage Publications.

Kachadourian, L. K., Fincham, F., y Davila, J. (2004). The tendency to forgive in dating and married couples: The role of attachment and 
relationship satisfaction. Personal Relationships, 11, 373-393.doi: 10.1111/j.1475-6811.2004.00088.x

Kishton, J. M., y Widaman, K. F. (1994). Unidimensional versus domain representative parceling of questionnaire items: An empirical example. Educational and Psychological Measurement, 54, 757-765. doi: 10.1177/0013164494054003022

Kline, R. B. (2005). Principles and practice of structural equation modeling $\left(2^{\text {nd }} E d\right.$.). New York: Guilford Press.

Kobak, R. R., y Sceery, A. (1988). Attachment in late adolescence: Working models, affect regulation, and representations of self and others. Child Development, 59, 135-146. doi: 10.2307/1130395

Lawler-Row, K. A., Younger, J. W., Piferi, R. L., y Jones, W. H. (2006). The role of adult attachment style in forgiveness following an interpersonal offense. Journal of Counseling and Development, 84, 493-502. doi: 10.1002/j.1556-6678.2006.tb00434.x

Little, T. D., Cunningham, W. A., Shahar, G., y Widaman, K. F. (2002). To parcel or not to parcel: Exploring the question, weighing the merits. Structural Equation Modeling: A Multidisciplinary Journal, 9, 151-173.

Luebbert, M. C. (2000). Attachment, psychological development, shame, guilt, and forgiveness, (Tesis de Doctorado no publicada), Texas A y M University, College Station, TX, Estados Unidos.

Makinen, J. A., y Johnson, S. M. (2006). Resolving attachment injuries in couples using emotionally focused therapy: Steps toward forgiveness and reconciliation. Journal of Consulting and Clinical Psychology, 74, 1055-1064. doi: 10.1037/0022006X.74.6.1055

Martínez, C., y Santelices, M. P. (2005). Evaluación del apego en el adulto: una revisión. Psykhe, 14, 181-191.doi: 10.4067/S071822282005000100014

McCullough, M. E., Rachal, K. C., Sandage, S. J., Worthington Jr., E. L., Brown, S. W., y Hight, T. L. (1998). Interpersonal forgiving in close relationships: II. Theoretical elaboration and measurement. Journal of Personality and Social Psychology, 75, 1586-1603.

McCullough, M. E., y Witvliet, C. V. O. (2002). The psychology of forgiveness. En C. R. Snyder y S. J. Lopez (Eds.), Handbook of positive psychology ( $2^{\text {nd }}$ ed., pp. 446-455). New York: Oxford University Press.

McCullough, M. E., Worthington, E. L., y Rachal, K. C. (1997). Interpersonal forgiving in close relationships. Journal of Personality and Social Psychology, 73, 321-336.

Mikulincer, M. (1998). Adult attachment style and affect regulation: Strategic variations in self-appraisals. Journal of Personality and Social Psychology, 75, 420-435. doi: 10.1037//0022-3514.75.2.420
Mikulincer, M., y Shaver, P. (2003). The attachment behavioral system in adulthood, psychodynamics, and interpersonal processes. En M. P. Zanna (Ed.), Advances in experimental social psychology (pp. 53-152). San Diego: Elsevier Academic Press.

Mikulincer, M., Shaver, P. R., y Pereg, D. (2003). Attachment theory and affect regulation: The dynamics, development, and cognitive consequences of attachment-related strategies. Motivation and Emotion, 27, 77-102. doi: 10.1023/A:1024515519160

Mikulincer, M., Shaver, P. R., y Slav, K. (2006). Attachment, mental representations of others, and gratitude and forgiveness in romantic relationships. En M. Mikulincer y G. S. Goodman (Eds.), Dynamics of romantic love. Attachment, caregiving, and sex (pp.190-215). New York: Guilford Press.

Mikulincer, M., Shaver, P. (2007). Attachment in adulthood: Structure, dynamics, and change. New York, NY: Guilford Press.

Paleari, F. G., Regalia, C., y Fincham, F. D. (2009). Measuring offencespecific forgiveness in marriage: The Marital Offence-Specific Forgiveness Scale (MOFS). Psychological Assessment, 21, 194-209. doi: $10.1037 / \mathrm{a} 0016068$

Pietromonaco, P. R., Greenwood, D., y Feldman, B. L. (2004). Conflict in adult close relationships: An attachment perspective. En W. S. Rohles y J. Simpson (Eds.), Adult attachment: Theory, research, and clinical implications (pp.267-299). New York: Guilford Publications.

Streiner, D. L. (2006). Building a better model: An introduction to Structural Equation Modeling. Canadian Journal of Psychiatry, 50, 115- 122.

Thompson, L. Y., Snyder, C. R., Hoffman, L., Michael, S. T, Rasmussen, H. N., ... Billings, L. S. (2005). Dispositional forgiveness of self, others, and situations. Journal of Personality, 73, 313-360. doi: 10.1111/j.14676494.2005.00311.x

Wang, T. W. (2008). Adult attachment and forgiveness in taiwanese college students. Psychological Reports, 103, 161-169. doi: 10.2466/ pr0.103.1.161-169

Webb, M., Call, S., Chickering, S. A., Colburn, T. A., y Heisler, D. (2006). Dispositional forgiveness and adult attachment styles. The Journal of Social Psychology, 146, 509-512. doi: 10.3200/SOCP.146.4.509-512

Wei, M., Vogel, D. L., Ku, T., y Zkalik, R. A. (2005). Adult attachment, affect regulation, negative mood, and interpersonal problems: The mediating roles of emotional reactivity and emotional cutoff. Journal of Counseling Psychology, 52, 14-24. doi: 10.1037/0022-0167.52.1.14 